\title{
Oil palm leaves and roots differ in physiological response, antioxidant enzyme activities and expression of stress-responsive genes upon exposure to drought stress
}

\begin{abstract}
The seedling stage is a critical period for survival under drought stress. To identify biochemical and molecular drought response changes, oil palm seedlings were exposed to different levels of drought severity. Total chlorophyll, total soluble protein and total proline content were measured while expression of stress responsive genes was quantified using qPCR. The diminishing total chlorophyll (chl) content and the ratio of $\operatorname{chl}_{\mathrm{a}}$ to $\operatorname{chl}_{\mathrm{b}}\left(\mathrm{chl}_{\mathrm{a}}\right.$ : $\left.\mathrm{chl}_{\mathrm{b}}\right)$ were observed to be significant. The significant reduction of $\mathrm{chl}_{\mathrm{a}}$ was closely related to photosystem II deficiency. Based on the effects of drought on chlorophyll content, the samples can be categorised into mild (7 days of water withholding; DWW), moderate (14 DWW) and severe (21, 28 and 35 DWW). Sample at 21 DWW was used to represent the severe stage. Genes encoding ethylene responsive binding protein, late embryogenesis abundant ( $L E A)$, dehydrin $(D H N)$, cold-induced, heat shock protein 70 and metallothionein type 2 were differentially up-regulated in the leaves, while in the roots only $L E A$ and $D H N$ were up-regulated. The proline content increased gradually in both vegetative tissues, while the total soluble protein content was affected by increasing drought severity. The activity of catalase was highest in the roots at the severe drought stage, while guaicol peroxidase activity was shown to be highest in the leaves under mild drought. These findings provide new insights into stress tolerance mechanisms of oil palm seedlings and can be used to develop stress tolerant oil palm through classical breeding and genetic engineering.
\end{abstract}

Keyword: Elaeis guineensis; Drought; Transcription factor; Stress responsive genes (SRGs); Enzymatic and non-enzymatic antioxidant; Vegetative tissues 